\title{
Signal transformation approach to fast nanopositioning
}

\author{
Abu Sebastian $^{1}$ and S. O. Reza Moheimani ${ }^{2}$ \\ ${ }^{1}$ IBM Zürich Research Laboratory, CH-8803 Rüschlikon, Switzerland \\ ${ }^{2}$ School of Electrical Engineering and Computer Science, The University of Newcastle, Callaghan, NSW 2308, \\ Australia
}

(Received 9 November 2008; accepted 3 June 2009; published online 6 July 2009)

\begin{abstract}
High bandwidth, high resolution positioning is a significant enabling tool for nanotechnology. Unfortunately, in a typical nanopositioner operating in closed loop, higher bandwidth results in increased sensitivity to measurement noise and hence reduced resolution. In this article we present a technique that allows high-precision tracking of repetitive, jitter-free reference signals without being constrained by the reduction in measurement noise induced positioning resolution. The technique works exceptionally well for scanners with high enough open loop bandwidth and in the absence of high frequency external disturbances. Both simulation and experimental results are presented to illustrate this concept using a triangular reference signal. () 2009 The American Physical Society. [DOI: 10.1063/1.3160016]
\end{abstract}

Precise positioning is the enabling technology for a variety of applications ranging from scanning probe microscopy and semiconductor manufacturing systems to data storage devices. ${ }^{1-3}$ Positioners such as piezoactuated flexure stages and Micro-Electro-Mechanical Systems (MEMS) microscanners are widely employed to perform fast scan operations in these devices. Significant effort has been devoted to the design of high bandwidth feedback controllers for nanopositioners. ${ }^{4,5}$ However feedback controllers make the closed-loop system susceptible to measurement noise. The positioning inaccuracies arising from the measurement noise tend to increase with increasing bandwidth of the closedloop system. At some point this has the potential to significantly impact the positioning accuracy thus defeating the purpose of using a highly precise positioner in the first place. Low bandwidth controllers on the other hand would not be able to track the high frequency components of the reference signals resulting in a highly undesirable tracking performance. This is a fundamental tradeoff that needs to be addressed while designing feedback controllers for nanopositioning systems.

Consider a one degree of freedom control configuration where $P$ denotes the model of the positioner and $K$ denotes the controller. Let the signals $r, x$, and $n$ denote the reference signal, the position of the scanner, and the measurement noise, respectively. The control objective is to have $x$ track $r$ using the measured position, $x_{m}$, which is $x$ corrupted by the measurement noise. The transfer function relating the position to the reference signal $T_{\mathrm{xr}}$ quantifies the extent to which the closed-loop system is able to track the reference signal. The transfer function relating the position to the measurement noise $T_{\mathrm{xn}}$ on the other hand quantifies the impact of measurement noise on positioning. These two transfer functions are given by

$$
T_{\mathrm{xr}}=-T_{\mathrm{xn}}=\frac{P K}{1+P K} .
$$

It can be seen that the magnitude response of $T_{\mathrm{xr}}$ is identical to that of $T_{\mathrm{xn}}$. This implies that an increased reference track- ing bandwidth invariably leads to a greater sensitivity to measurement noise.

A common approach employed to circumvent this tradeoff is to reduce the high frequency content of the reference signal without substantially distorting it. ${ }^{6}$ However there are always limits to which the distortions to the original reference signal can be tolerated. For example in a triangular reference signal, reducing the high frequency components would substantially reduce the available linear region. Another approach is to design higher order controllers. Even in this approach only partial decoupling of the reference tracking and noise sensitivity transfer functions is possible and moreover, this approach results in significantly more complicated controllers. In this article we propose a novel scheme that tackles the problem of tracking repetitive reference signals. The essential idea is to transform the signals within the feedback loop so that the tracking problem can be reduced to that of tracking an alternate reference signal with much lower frequency content.

The basic idea is to find a transformation and inverse transformation between the repetitive reference signal and a dummy reference signal with much less frequency content. For example one can find a map that transforms a repetitive triangular reference signal into a ramp signal with a constant slope. The next step is to design a controller that tracks the dummy reference signal with zero steady state error. In Fig. 1, a block diagram depicting the signal transformation scheme is presented. The mapping relating the dummy reference signal with the actual reference signal is denoted by $\Phi$ and the inverse mapping is denoted by $\Phi^{-1}$.

The map $\Phi$ which corresponds to a triangular reference signal is shown in Fig. 2. Let $i$ denote the index of half periods of a triangular reference signal. If $t$ denotes the time and $T$ denotes the period of the triangular signal, then $i$ is given by $i=\lfloor 2 t / T\rfloor+1$, where $\lfloor$.$\rfloor denotes the floor operator. If$ $A$ denotes the amplitude of the triangular signal, then $\Phi$ is given by 


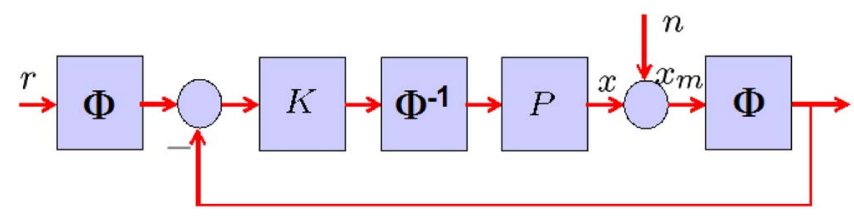

FIG. 1. (Color online) Block diagram depicting the signal transformation based control scheme.

$$
y=\Phi(x)=(-1)^{(i-1)} x(t)+2 A\lfloor i / 2\rfloor,
$$

and $\Phi^{-1}$ is given by

$$
y=\Phi^{-1}(x)=(-1)^{(i-1)} x(t)+(-1)^{i} 2 A\lfloor i / 2\rfloor .
$$

These functions, $\Phi$ and $\Phi^{-1}$ are specifically chosen so that they translate the signals in the frequency domain. $\Phi^{-1}$ transformation ahead of the scanner $P$ ensures that the scanner sees a signal close to a triangular signal and the $\Phi$ transformation on the measured position signal ensures that the controller sees a signal close to a ramp signal.

The controller $K$ is chosen such that the closed-loop system tracks the dummy reference signal, ramp signal in this case, with zero steady state error. Given that the reference signal is a repetitive triangular signal, the dummy reference signal is a ramp signal with a constant slope. To track a ramp signal with zero steady state error we require a type 2 system and hence $K$ should have a double integral component. So a possible control structure could be, $K(s)=k_{i} / s+k_{i i} / s^{2}$, where $k_{i}$ and $k_{i i}$ are integral and double integral gains.

The essential idea is that, owing to the blocks $\Phi$ and $\Phi^{-1}$ within the feedback loop, the problem of tracking a triangular signal (with high frequency content) is translated to that of tracking a ramp signal (with low frequency content). This way from the perspective of the closed-loop system it would be tracking a signal close to a ramp signal, which it can track satisfactorily in the steady state irrespective of the slope of the ramp signal. However, from the perspective of the scanner, it will be tracking a signal close to a triangular signal.

Triangular signals are widely employed in a variety of nanopositioning applications for the fast axis in an $X-Y$ nanopositioner. Hence we focus on applying the signal transformation approach to tracking triangular reference signals. However, it could also be extended to other reference signals if the appropriate $\Phi$ and $\Phi^{-1}$ can be obtained.

Simulation results are presented to demonstrate the effectiveness of the scheme. In the case where $P$ can be approximated by a constant gain, irrespective of the frequency of the triangular reference signal, the closed loop system asymptotically tracks the reference signal with a conver-

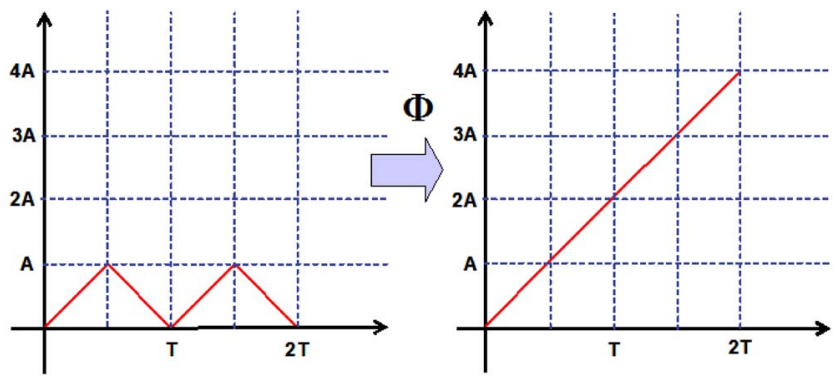

FIG. 2. (Color online) The map $\Phi$ transforms a triangular signal to a ramp signal.

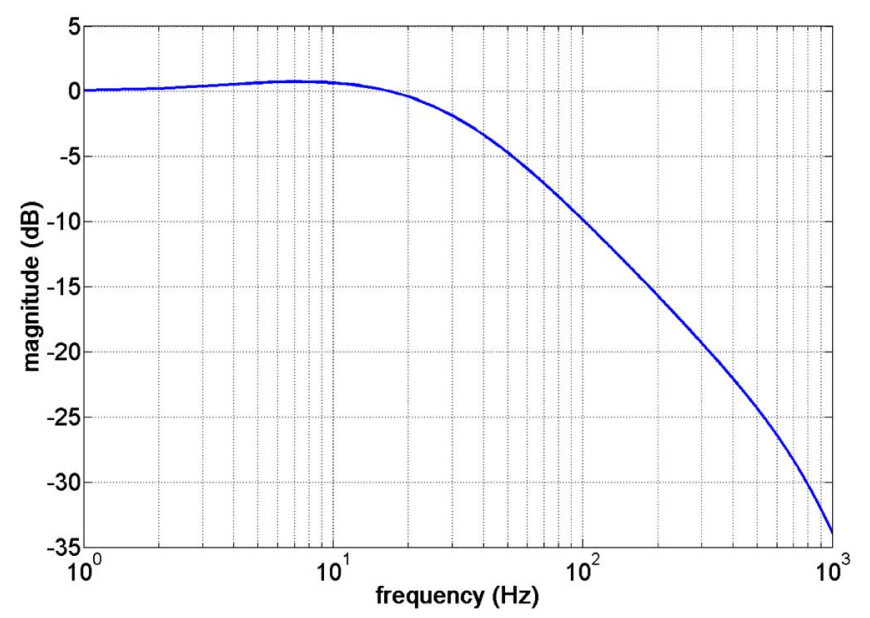

FIG. 3. (Color online) The magnitude response of transfer function $T_{\mathrm{xr}}$ which is also identical to that of $T_{\mathrm{xn}}$.

gence time given by the time it takes for the closed loop system to track the corresponding ramp signal. Deviations from a constant gain behavior would deteriorate the tracking performance. The proposed controller will be incapable of correcting for any higher frequency motion of the scanner excited by the triangular signal. Most scanners used in nanopositioning applications are well modeled by a damped harmonic oscillator model with the possible addition of higher order resonances. So it is essential that the open loop scanner resonances are significantly higher than the frequency of the triangular signal being tracked and that they are well damped. This is not a significant limitation as most of the commercial scanners have the first resonance frequency in excess of $500 \mathrm{~Hz}$. Significant research has gone into having the first resonance frequency as high as possible for both piezoactuated scanners and MEMS microscanners. ${ }^{7,8}$ There also exist a number of active $e^{9}$ and passive ${ }^{10}$ schemes to damp the first resonance, which are marginally sensitive to measurement noise. Note that these requirements are on the open loop dynamics of the scanner. The closed-loop system can have very low bandwidth and hence very low sensitivity to measurement noise.

For simulation purposes a damped harmonic oscillator model is chosen with resonance frequency of $1 \mathrm{kHz}$ and quality factor of 1 . The model $P$ used for the simulation is given by $P(s)=3.948 \times 10^{7} /\left(s^{2}+6283 s+3.948 \times 10^{7}\right)$ and a double integral controller $K$ is designed given by $K(s)=200 / s$ $+5000 / s^{2}$. The closed-loop transfer function relating the position with the reference, $T_{\mathrm{xr}}$, which is identical to the transfer function relating the position with the measurement noise, $T_{\mathrm{xn}}$, is shown in Fig. 3. The scan performance of the closed-loop system is shown in Fig. 4. A $100 \mathrm{~Hz}$ triangular signal is tracked with and without the scan signal transformation scheme. Note that since $T_{\mathrm{xr}}$ has a $-3 \mathrm{~dB}$ bandwidth of only $35 \mathrm{~Hz}$, it is hardly possible to track a triangular signal of fundamental frequency at $100 \mathrm{~Hz}$. However with the signal transformation approach within a few cycles, the closedloop system is able to track the $100 \mathrm{~Hz}$ reference signal. Figure 4 also shows the tracking performance after $0.2 \mathrm{~s}$. With the signal transformation scheme, the controller with a tracking bandwidth of just $35 \mathrm{~Hz}$ is able to track the $100 \mathrm{~Hz}$ triangular signal remarkably well. Since the noise sensitivity 

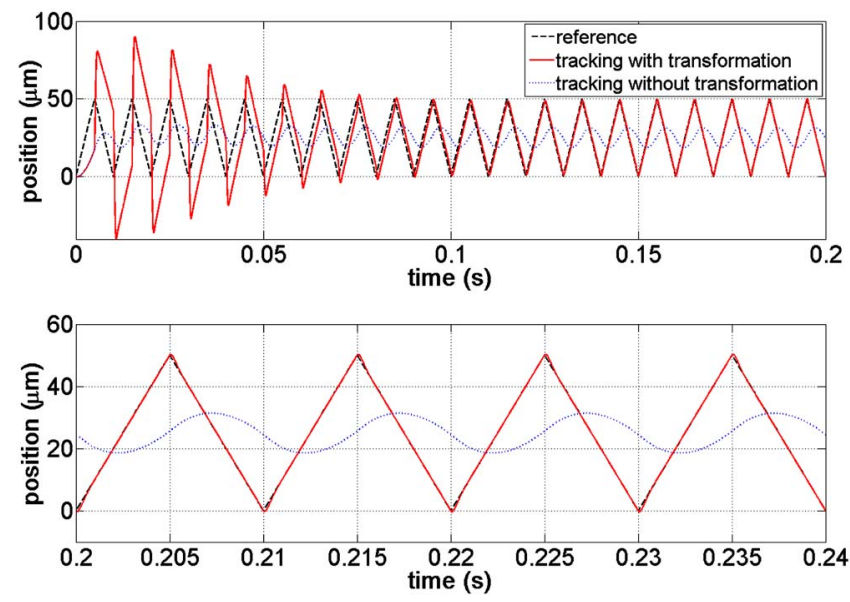

FIG. 4. (Color online) The scan performance while tracking a $100 \mathrm{~Hz}$ triangular signal (top) and steady state scan performance (bottom).

transfer function is identical to the reference tracking bandwidth, the closed-loop is equally less sensitive to measurement noise. However note that the system's sensitivity to input and output disturbances and actuator noise will be high if the closed-loop bandwidth is low. However, in many nanopositioning applications, because the systems are operated under controlled environmental conditions, the sensitivity to external disturbances is not a critical aspect. We present some experimental results on a commercial nanopositioner to further illustrate the merit of the scheme.

Experimental results obtained from a commercial nanopositioner are presented here. The nanopositioner is P517 from Physik Instrumente with built-in $X-Y-Z$ motion capability. We demonstrate the control of the scanner along the $X$-scan direction. As mentioned previously, one would like to have a scanner which has linear dynamic behavior and sufficient damping for the scheme to work satisfactorily. We had to shape the scanner dynamics such that these characteristics were met. To avoid nonlinearities arising from hysteresis, a charge amplifier (see Ref. 11) was used to drive the scanner. A resonant controller ${ }^{9}$ was used to damp the nanopositioner's first resonance. Note that this damping could also be achieved using sensor-less passive damping schemes such as the ones described in Ref. 10. Either way the damping mechanisms are marginally sensitive to measurement noise. A controller was designed such that the magnitude response of $T_{\mathrm{xr}}$ is given by Fig. 5 (top). The $-3 \mathrm{~dB}$ bandwidth is approximately $15 \mathrm{~Hz}$. A $20 \mathrm{~Hz}$ triangular reference signal is tracked using this controller and the signal transformation
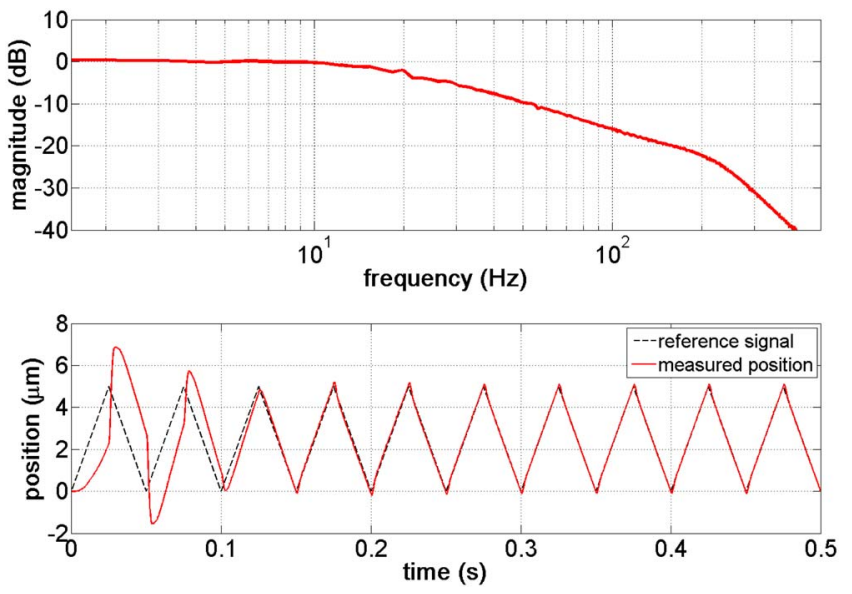

FIG. 5. (Color online) The experimentally measured magnitude response of the transfer function $T_{\mathrm{xr}}$ (top) and the scan performance while tracking a 20 $\mathrm{Hz}$ triangular signal (bottom).

scheme. As seen in Fig. 5, the system is able to track the 20 $\mathrm{Hz}$ reference signal within a few cycles. This experiment clearly demonstrates the efficacy of the new approach in a real experimental system.

We would like to thank Angeliki Pantazi for fruitful discussions and P. Bächtold for the design of the electronics used in this work. Special thanks go to Haris Pozidis and Evangelos Eleftheriou for their support of this work.

${ }^{1}$ S. Mishra, J. Coaplen, and M. Tomizuka, IEEE Control Syst. Mag. 27, 20 (2007).

${ }^{2}$ A. Sebastian, A. Pantazi, H. Pozidis, and E. Eleftheriou, IEEE Control Syst. Mag. 28, 26 (2008).

${ }^{3}$ S. Devasia, E. Eleftheriou, and S. O. R. Moheimani, IEEE Trans. Control Syst. Technol. 15, 802 (2007).

${ }^{4}$ G. Schitter, P. Menold, H. F. Knapp, F. Allgower, and A. Stemmer, Rev. Sci. Instrum. 72, 3320 (2001)

${ }^{5}$ S. Salapaka, A. Sebastian, J. P. Cleveland, and M. V. Salapaka, Rev. Sci. Instrum. 73, 3232 (2002).

${ }^{6}$ A. Fleming and A. G. Wills, Proceedings of the IFAC World Congress, Seoul, Korea, 2008 (unpublished), pp. 11805-11810.

${ }^{7}$ T. Ando, N. Kodera, E. Takai, D. Maruyama, K. Saito, and A. Toda, Proc. Natl. Acad. Sci. U.S.A. 98, 12468 (2001).

${ }^{8}$ G. Schitter, K. J. Aström, B. E. DeMartini, P. J. Thurner, K. L. Turner, and P. K. Hansma, IEEE Trans. Control Syst. Technol. 15, 906 (2007).

${ }^{9}$ S. O. R. Moheimani and B. J. G. Vautier, IEEE Trans. Control Syst. Technol. 13, 1021 (2005).

${ }^{10}$ A. J. Fleming and S. O. R. Moheimani, IEEE Trans. Control Syst. Technol. 14, 33 (2006).

${ }^{11}$ A. J. Fleming and S. O. R. Moheimani, Rev. Sci. Instrum. 76, 073707 (2005). 